\title{
Possibly preventable cardiac arrest in a morbidly obese patient - a comment on the 2015 ERC guidelines
}

Felix Patricius Hans ${ }^{\text {* }}$, Claudia Johanna Maria Hoeren², Phillipp Kellmeyer ${ }^{3}$, Lisa Hohloch ${ }^{1}$,Hans-Jörg Busch ${ }^{1}$ and Jörg Bayer ${ }^{1}$

\begin{abstract}
Background: The incidence of overweight and obesity has been steadily on the rise and has reached epidemic proportions in various countries and this represents a well-known major health problem. Nevertheless, current guidelines for resuscitation do not include special sequences of action in this subset of patients. The aim of this letter is to bring this controversy into focus and to suggest alterations of the known standard cardiopulmonary resuscitation in the obese.
\end{abstract}

Case presentation: An obese patient weighing $272 \mathrm{~kg}$ fell to the floor, afterwards being unable to get up again. Thus, emergency services were called for assistance. There were no signs or symptoms signifying that the person had been harmed in consequence of the fall. Only when brought into a supine position the patient suffered an immediate cardiac arrest. Cardiopulmonary resuscitation was performed but there was no return of a stable spontaneous circulation until the patient was brought into a full lateral position. In spite of immediate emergency care the patient ultimately suffered a lethal hypoxic brain damage.

Conclusion: A full lateral position should be considered in obese patients having a cardiac arrest as it might help to re-establish stable circulatory conditions.

Keywords: Cardiopulmonary resuscitation, Heart arrest, Obesity, Emergencies, Emergency medical services, Emergency service, Hospital

\section{Letter to the Editor}

Increasing numbers of overweight and obese patients require tailored therapeutic approaches to diseases that come along with such alterations of human constitution and metabolism [1]. Especially the acute care and emergency treatment of this subset of patients is challenging and needs to be constantly adapted. Although recently updated, guidelines for cardiopulmonary resuscitation do not mirror those changes in prevalence as no practical instructions are provided to handle morbidly obese patients $[2,3]$. We would like to ad a comment to the latest guidelines in the form of a case report, in order to

\footnotetext{
* Correspondence: felix.hans@uniklinik-freiburg.de

${ }^{1}$ University Emergency Department, Medical Center - University of Freiburg,

Sir-Hans-A-Krebs-Strasse, 79106 Freiburg, Germany

Full list of author information is available at the end of the article
}

draw attention to this topic and to give a lead in treatment of those patients.

\section{Case presentation}

A 60-year-old patient fell to the floor inside his apartment and alarmed emergency medical services (EMS) consequently. Due to his estimated body mass of $>250 \mathrm{~kg}$ the patient was unable to get to his feet again by himself. On arrival of the EMS the alert patient lay in a right lateral position and reported no pain, no dyspnea, no chest tightness or any other symptoms suspicious for an acute medical condition or injury. Up to this point, the only disease (apart from obesity) the patient had to deal with was a gonarthrosis. Having experienced such a situation before the patient told the EMS to lift him up again with the help of a "board". A fire truck was sent to support the EMS team and a backboard was installed to lift the 
patient. No medical equipment was needed at that time. The moment the patient was moved onto the backboard and brought into a supine position he gasped, his face went cyanotic and he lost consciousness. Without palpable pulse the patient was immediately resuscitated. Cardiopulmonary resuscitation (CPR) was alternately performed by six trained firemen and two paramedics. Medical equipment was installed to support CPR and an emergency physician was called, following the locally implemented protocol. According to the guidelines, the detected asystole was treated by application of epinephrine and the airway was secured by a supraglottic airway device. Return of spontaneous circulation (ROSC) was rapidly achieved consecutively for three times with the ECG showing an atrial fibrillation each time spontaneous circulation returned. Pulses were palpable but the heart rhythm then rapidly degenerated into brady-asystole. The EMS suspected an aorto-caval compression causing the brady-asystole and turned the patient into a full lateral position after the subsequent ROSC - a measure which lead to the patient's hemodynamic stabilization and allowed transportation to the cardiac arrest center. During the initial clinical workup, a pulmonary embolism was ruled out by CT-scan, laboratory findings revealed a mild myocardial necrosis (interpreted as myocardial infarction type II as there were no regional cardiac wall motion abnormalities). Coronary angiography could not be performed due to the patient's weight and size. Therapeutic hypothermia was not performed as none of the available systems were considered applicable. During the intensive care stay the patient was transferred in a half-sitting position and remained hemodynamically stable.

The actual patient's weight proved to be $272 \mathrm{~kg}$. Aortocaval compression could not be reproduced (by ultrasound) under invasive ventilation conditions but was assumed to be the most likely cause of the cardiac arrest. Stimulation of the vagus nerve by the large abdominal mass was considered another potential mechanism for the brady-asystole.

Unfortunately, the patient suffered a hypoxic brain damage to such an extent that he never gained consciousness again and died 5 days after hospital admission.

\section{Discussion}

In this case, a setting - which at first glance seemed to be rather trivial and easy to handle - led to a fatal patient outcome despite instantly initiated high quality CPR by well-trained staff and treatment in a cardiac arrest center. Although never clearly proven, the circumstances mentioned indicate that an aortocaval compression syndrome was the cause of the hear arrest. Morbidly obese patients should therefore be treated with precaution, always bearing in mind the possible physical impact of the patient's body mass. Based on our experience in the above described CPR setting, especially in PEA/asystole with inefficient chest compressions or rapidly terminating ROSC in the morbidly obese, a tilt maneuver might be considered. With $15^{\circ}$ tilts having proven to be ineffective in similar situations we suggest a minimum of $30^{\circ}$ to a full lateral tilt as an measure to maintain stable circulatory conditions $[4,5]$.

\section{Conclusion}

A full lateral position should be considered in obese patients having a cardiac arrest as it might help to reestablish stable circulatory conditions.

\section{Abbreviations}

CPR: Cardiopulmonary resuscitation; CT: Computer tomography; ECG: Electrocardiogram; EMS: Emergency medical services; KG: Kilogram; PEA: Pulseless electric activity; ROSC: Return of spontaneous circulation

\section{Acknowledgements}

The article processing charge was funded by the German Research Foundation (DFG) and the Albert Ludwigs University Freiburg in the funding programme Open Access Publishing.

\section{Funding}

None.

Availability of data and material

All clinical data is available, including radiographs and labworks.

Authors' contributions

All authors were involved in the clinical treatment of the patient or the preparation of this manuscript.

Competing interests

The authors declare that they have no competing interests.

\section{Consent for publication}

The next of kin, the patient's brother, gave written consent to the publication in the above manner.

\section{Ethics approval and consent to participate}

Ethics approval was not necessary as all patient data has been anonymized (Application Number 10013/16 from July 11th 2016, Ethics commission of the Alber-Ludwings- University Freiburg, Engelbergerstrasse 21, 79106 Freiburg. Response from July 22nd: No ethics approval needed).

\section{Author details}

${ }^{1}$ University Emergency Department, Medical Center - University of Freiburg, Sir-Hans-A-Krebs-Strasse, 79106 Freiburg, Germany. ${ }^{2}$ Department of Anesthesiology and Critical Care, Medical Center - University of Freiburg, Hugstetter Strasse 55, 79106 Freiburg, Germany. ${ }^{3}$ Department of Neurology and Neuroscience, Medical Center - University of Freiburg, Breisacher Str. 64, 79106 Freiburg, Germany.

Received: 3 August 2016 Accepted: 14 September 2016 Published online: 04 October 2016

References

1. World Health Organization. Obesity and overweight. http://www.who.int/ mediacentre/factsheets/fs311/en/. Accessed 4 Jul 2016.

2. Lavonas EJ, Drennan IR, Gabrielli A, Heffner AC, Hoyte CO, Orkin AM, et al. Part 10: Special Circumstances of Resuscitation. Circulation. 2015;132:S501-18. doi:10.1161/CIR.0000000000000264.

3. Truhlář A, Deakin CD, Soar J, Khalifa GEA, Alfonzo A, Bierens JJ, et al. European Resuscitation Council Guidelines for Resuscitation 2015: Section 4. Cardiac arrest in special circumstances. Resuscitation. 2015;95:148-201. doi: 10.1016/j.resuscitation.2015.07.017. 
4. Higuchi H, Takagi S, Zhang K, Furui I, Ozaki M. Effect of lateral tilt angle on the volume of the abdominal aorta and inferior vena cava in pregnant and nonpregnant women determined by magnetic resonance imaging. Anesthesiology. 2015:122:286-93. doi:10.1097/ALN.0000000000000553.

5. Lee SWY, Khaw KS, Ngan Kee WD, Leung TY, Critchley LAH. Haemodynamic effects from aortocaval compression at different angles of lateral tilt in non-labouring term pregnant women. Br J Anaesth. 2012;109:950-6. doi: 10.1093/bja/aes349

Submit your next manuscript to BioMed Central and we will help you at every step:

- We accept pre-submission inquiries

- Our selector tool helps you to find the most relevant journal

- We provide round the clock customer support

- Convenient online submission

- Thorough peer review

- Inclusion in PubMed and all major indexing services

- Maximum visibility for your research

Submit your manuscript at www.biomedcentral.com/submit 